Richand Rnomland= Solli Istad

Kristoffer Jensen I
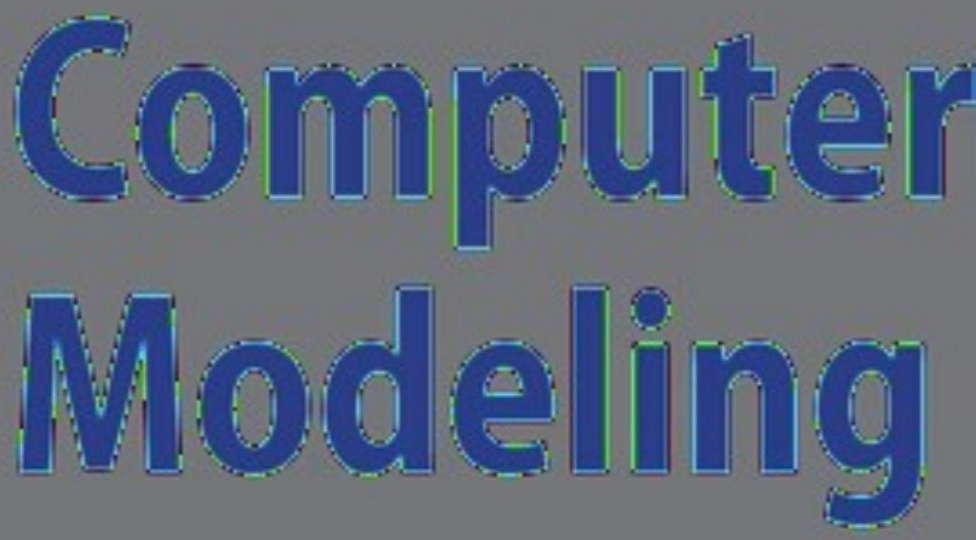

Sense of Sounds

4th International Symposit Copenhagen, Denmark, Au Revised Papers 


\title{
DECONcert: Making Waves with Water, EEG, and Music
}

\author{
Steve Mann ${ }^{1}$, James Fung ${ }^{1}$, and Ariel Garten ${ }^{2}$ \\ ${ }^{1}$ University of Toronto \\ Dept. of Electrical and Computer Engineering \\ Toronto, Ontario, Canada \\ ${ }^{2}$ Neuroconsulting \\ Toronto, Ontario, Canada
}

\begin{abstract}
We describe events in which music, water and the brain form an immersive environment for human-computer and human-computerhuman collective engagement. The theme of sound wave production, regeneration and audition from water waves and brain waves is our central exploration, beginning with our DECONcerts in which participants, immersed in water and connected to EEG equipment, regeneratively create or affect live music by varying their alpha wave output. We explored the five states-of-matter (Classical Elements) of solid ("Earth"), liquid ("Water"), gas ("Air"), plasma ("Fire"), and quintessence ("Idea"), in the context of immersive media (e.g. when the surrounding state-of-matter was liquid). Some of these immersive environments spanned multiple countries, by way of networked connectivity. We also expanded from philosophical to therapeutic contexts by including Parkinson's patients in our immersed environments.
\end{abstract}

\section{Introduction}

This paper presents a series of performances, art exhibits, and concerts, that explored the relationships between water waves, sound waves, and brainwaves (see figure (1I). These events merged a custom built EEG (Electroencephelograph or "brainwave") computational system with music generation, immersive aquatic spaces, and groups of immersively engaged performers and participatory audiences. Participatory performances explored collective consciousness by creating both physically shared spaces (connecting various groups of participants across distant geographical boundaries) and shared human-computational networks.

The resulting collective immersive experiences were created using the media of a shared immersive audio environment, and an aquatic environment where, in some events, groups of participants and performers were actually immersed, in whole, or in part, in water. These media are explorations of waves: in one medium, acoustic waves; in another, aqueous waves (various performances studied caustics and wavefronts, as well as water-induced sounds); and, of course, brainwaves. 


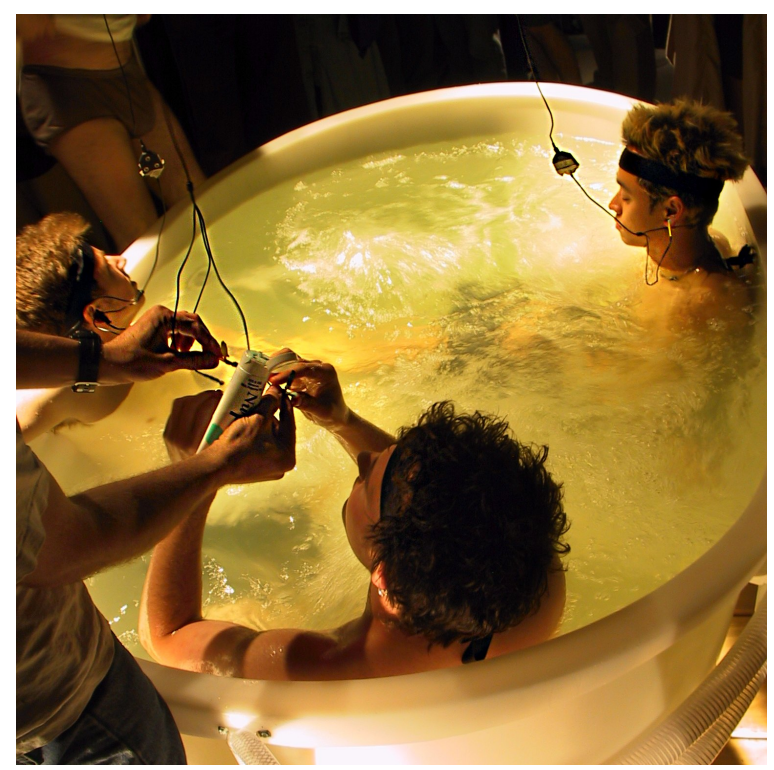

(a)

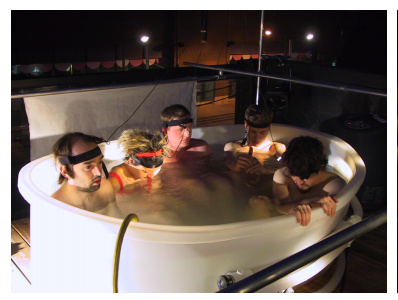

(b)

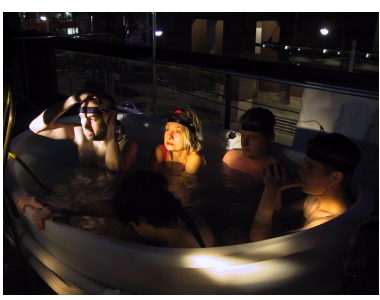

(c)

Fig. 1. DECONcert events allowed participants to explore issues of DECONtamination, music, water, and brainwave. (a) Participants being prepared with electrode paste for EEG readings. (b,c) A separate spotlight on each participant responds to their individual level of visual arousal, thus turning participants into performers who are on stage in the bath.

This theme of waves not only occurred at the observational (output) side of the performance, but also at the input: both the ambient and acoustic environments were generated by directly measuring and interpreting the brainwaves of the participants. This paper discusses various performance art events together with the philosophical implications and artistic narratives developed in the various events.

\section{Creating Immersive Experiences with Humanistic Intelligence}

Humanistic intelligence (HI) is defined [7] as a signal processing framework in which the processing apparatus is inextricably intertwined with the natural 
capabilities of our human body and mind. Within the processing framework of HI, the computational apparatus and user, in being intertwined as such, are considered as a signal processing block, interacting as one with the outside world. Within this block, the human and computer work together in a tight feedback loop, with each accepting information and outputs from, and providing information and inputs to, each other. In contrast to AI, which seeks to recreate human intelligence on the machine, HI seeks to utilize the abilities of both the human and machine to their fullest.

HI forms the structural framework in our explorations. In each of our exploratory performances, exhibits, and concerts, water, music and brainwaves, and states-of-matter become the media to express Humanistic Intelligence. Though at first inspection the HI framework may appear to express an individual's relationship to computational apparatus, our events demonstrate the collective nature of HI, where groups of participants are all connected to the computational system and thus to each other: a collective consciousness.

We use music and water and brainwaves as mediums to create group immersion to allow participants to experience and become a collective consciousness. We explore collective consciousness through: (1) collective consciousness (i.e. using multiple participant brainwave inputs to drive an artistic process), (2) representing these brainwave signals in a shared multimedia environment where audiovisual experiences, such as sound and visuals, are collectively experienced; and (3) using water as a physical agent to bring participants into a shared space that is truly, and literally immersive (in the sense of a communal bathing experience). These media explore issues of privacy and personal space.

\section{The Events}

Starting in July 20011 we had a series of events addressing issues of contamination and biological warfare. The authors created a number of events, performances, and concerts that dealt with issues surrounding decontamination. These events were named DECONference, DECONversation, DECONsortium, DECONtrol, DECONcert, and the like, making reference to DECONtamination. For example, a series of DECONcerts were presented as DECONtamination concerts in which participants were washed down with water prior to being connected to EEG (brainwave) instrumentation.

Our DECONcert series explored a regenerative feedback loop between brainwaves and music, as the collective consciousness of a large audience either generated music or modified music generated by other performers.

In our Powerplant 2 DEConcert, individuals contributed directly by playing their brain as an instrument in an improvising live band, directing and taking direction from more traditional musical interactions.

\footnotetext{
${ }^{1}$ Our first event took place prior to the anthrax scare that came shortly after the September 11th 2001 terrorist attacks.

2 The "Powerplant" is a Canadian contemporary art gallery.
} 
In many of our DECONcerts, groups of people from around the world were connected, over the Internet, from various different communal baths or aquatic spaces. For example, in one DECONcert, we had groups of six bathers, at a time, in one rooftop tub, each outfitted with EEG electrodes, connected to bathers, three-at-a-time, in another distant tub that was located on the sidewalk of a busy downtown street. Situating the bath on a busy sidewalk established a juxtaposition of public and private, while inviting passers-by to stop, "doff their duds", put on the EEG electrodes, and join in. The different group baths were connected audiovisually, as well as electroencephalically (using EEG sensors), across the World Wide Web, also by way of web cameras, microphones, and various physiological signals such as EEG and ECG (Electrocardiogram).

In another concert, we invited a number of Parkinson's patients to participate remotely from their hospital beds using equipment we sent out on loan. What was remarkable about this form of participation, was the fact that the DECONcerts were inclusive for people of any physical ability. In this form of cyborg space, a person of lesser physical ability is still a full participant, since the primary experimental control modality is brainwaves. All that is needed to be a full participant is a sufficiently engageable brain.

An important artistic narrative was the juxtaposition of this corporeal transcendence, combined with the physicality of passers-by in their disrobed and electrified bodies, situated in a bath on a busy street.

One set of mini-concerts within the DECONcert series was called "Telematic Tubs Against Terror". This was a series of events in which groups of individuals were immersed in tubs of water and connected by way of EEG to form a collective consciousness. This emerged as participants projected a sub-collective from each tub (each "wash node"). These events explored a collective and distributed consciousness as people's brainwaves were made public, in concert with the collectively shared experience of water and music.

Water and music also formed the backbone of our concert at the International Computer Music Conference (ICMC). The creation of a novel instrument called the hydraulophone, whose sound production is created using water as a medium, invited the examination of other mediums in which sound could be produced. Thus we created a physics-based orgonology in which musical instruments are classified based on the state-of-matter (solid, liquid, gas, plasma, or quintessence) of the intial sound-producing mechanism. We already created instruments from non-matter, i.e. quintessence (bio)informatics, e.g. brainwaves.

\subsection{DECONcert: Collaborative Music in the Key of EEG}

DECONcerts were a form of audience-participatory concert in which the participants' brainwaves determined the music they were experiencing.

The first DECONcert was, to the authors' knowledge, the first exploration of music generated by collective consciousness (i.e. more than one person generating music with their brainwaves together).

Our first collectively created concert, DECONcert 1, attracted enough interest to require three separate sessions in the same evening, each for a different group 


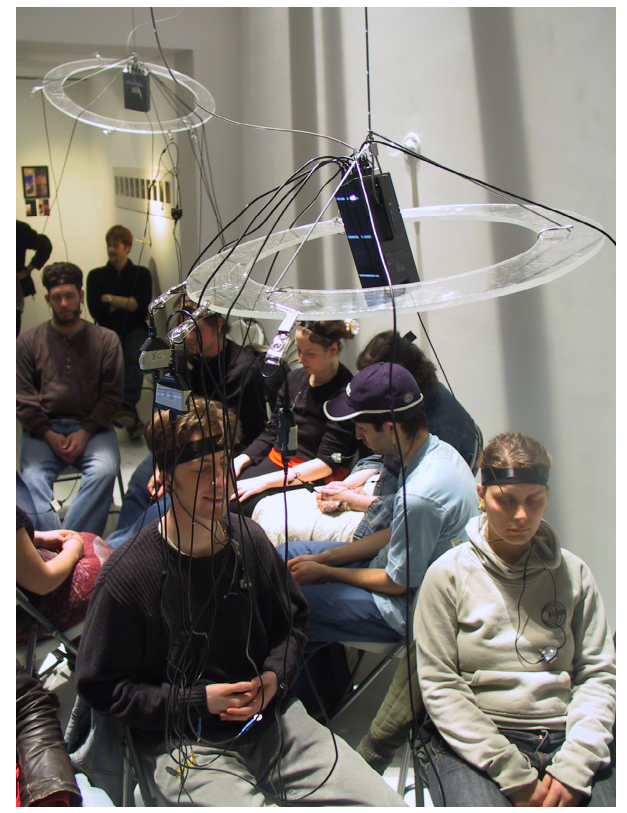

(a)

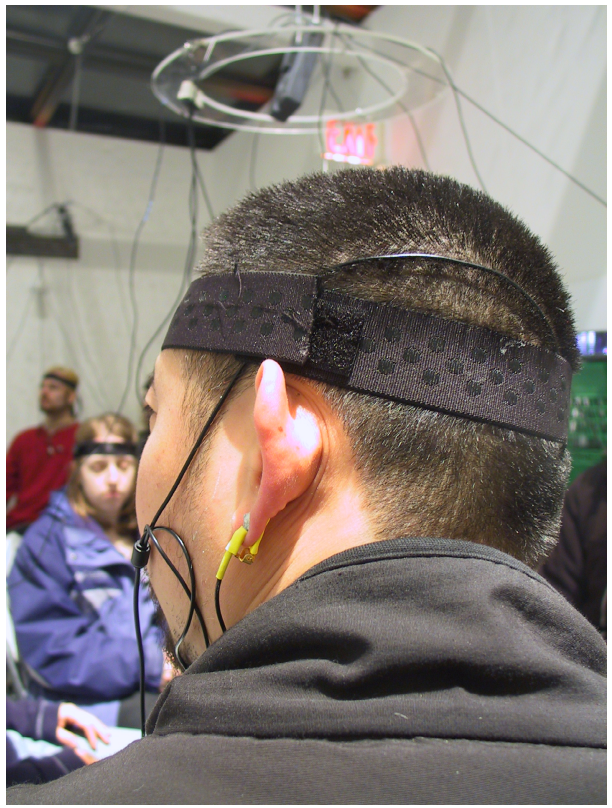

(b)

Fig. 2. DECONcert Performance: This group of 48 participants simultaneously and collectively adjusted the musical environment with their brainwaves while remotely connected to groups in other countries

of participants. For each session, we connected 48 people by way of their EEG signals, which were collectively used to affect the audiovisual environment.

Using six 8-person EEG machines, donated by manufacturer Thought Technologies Limited, we were able to obtain connections from 48 people at the same time.

In order to have the greatest flexibility we wrote our own GNU/Linux device drivers for these machines, and we developed and implemented our own signal processing algorithms. We developed a system to utilize multiple EEG signals to clean the signal and look for collective alpha synchronization (which occurs, for instance, when people close their eyes). Figure 2 shows images taken of the first DECONcert performance.

DECONcert utilized electroencephalogram (EEG) sensors which sensed electrical activity produced in the brains of the participants. The signals from the brainwaves of the 48 participants were used as input to dynamically alter a computationally controlled soundscape. DECONcert allowed the participants to form a feedback loop with the computational process of musical composition. The soundscape being generated was in response to the participants: the collective response from the group of participants is sensed by the computer, which then alters the music based upon this response. Again, the participants hear the music, and again respond, and again the computer senses and further alters the 
sound. In this way, collaborative biofeedback is being used in conjunction with an intelligent signal processing system to continually re-generate the music on the fly.

A total of 3 DECONcerts were held with different configurations exploring different methods of audience interaction. In each of the 3 DECONcerts that were held, up to 48 audience members sat in front of the stage in 6 groups. On stage, jazz musicians improvised on some combination of electric keyboard, electric clarinet, trumpet, saxophone, drums, and/ or base. As audience members listened to the concert, each member's brainstate determined the modulation of the output of the musician's synthesized instruments. Some acoustic qualities that the audience was able to modulate included pitch, volume, FM oscillation, chorus, and distortion. Figure 3 shows images from DECONcerts 2 and 3.

The participant's raw EEG signal, and frequency distribution, were plotted and projected onto a screen, so the participant could determine if he or she was in an alpha brain state or a beta state. When all of the participants in a single group reached alpha frequency (as determined by an averaging process), the acoustic quality controlled by that group was modulated accordingly.

In this way, the participants' brainwaves collectively and continuously affect music that was being heard. This process was both fluid and regenerative, in that participants' brain states influenced the musical output, which in turn was received (heard) by the participant's brain, which then influenced the participant's brainstate which influenced the music output.

\subsection{Powerplant Performance}

In 2007, we undertook a new iteration of the brainwave musical interface system. Rather than creating a feedback interaction between performer and audience, we allowed participants to "jam along" with a renowned live improvising band in a concert setting. We assigned each participant a note or chord. By reaching a certain threshold of alpha activity (20\% of total brainwave output measured from occipital lobe) participants were able to increase the volume of that tone. The increase was cumulative (temporally integrated) whereby the longer the participant remained over threshold, the higher the volume went. This cumulative (integrated) response mimics the response of aquatic instruments like the hydraulophone, which responds to absement or presement (time time-integral of displacement or reciprocal displacement) rather than to displacement or to velocity (many other polyphonic instruments like the piano respond to velocity rather than displacement or absement). This kind of response simulates the effect of a water reservoir that fills up or empties out over time, giving the instrument's user-interface an aquatic feel.

Once participants learned, through usage, how to gain control over the system, they could play and mute, warp and vibrate their note, in time with the band. Not only did the participants play along, but they also gave musical cues to the other players. This is a direct example of a humanistic intelligence signaling block, where human and machine work together directly affecting and responding to one another's output as it becomes reified in the external physical world. See Fig G 


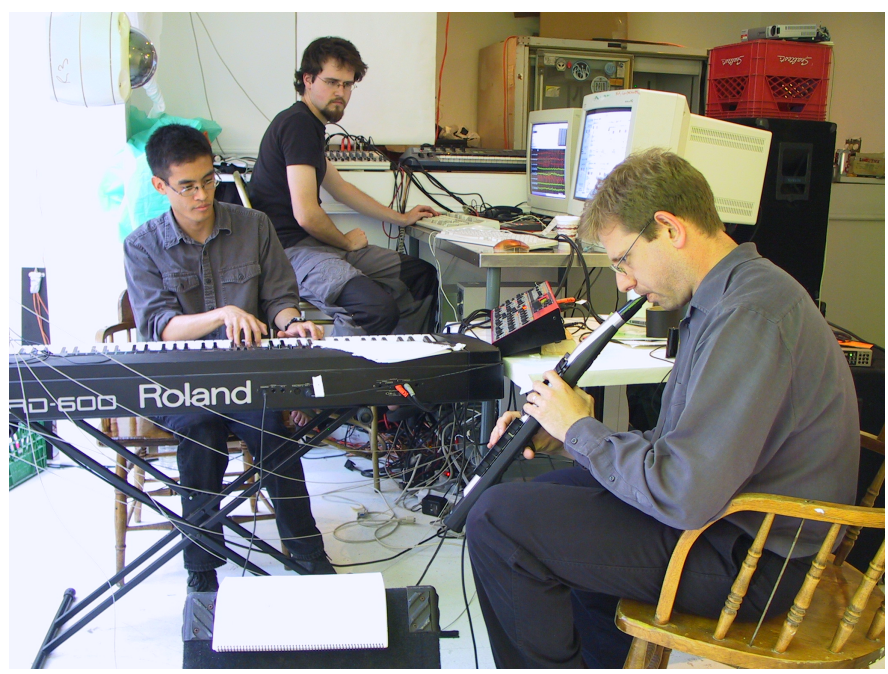

(a)

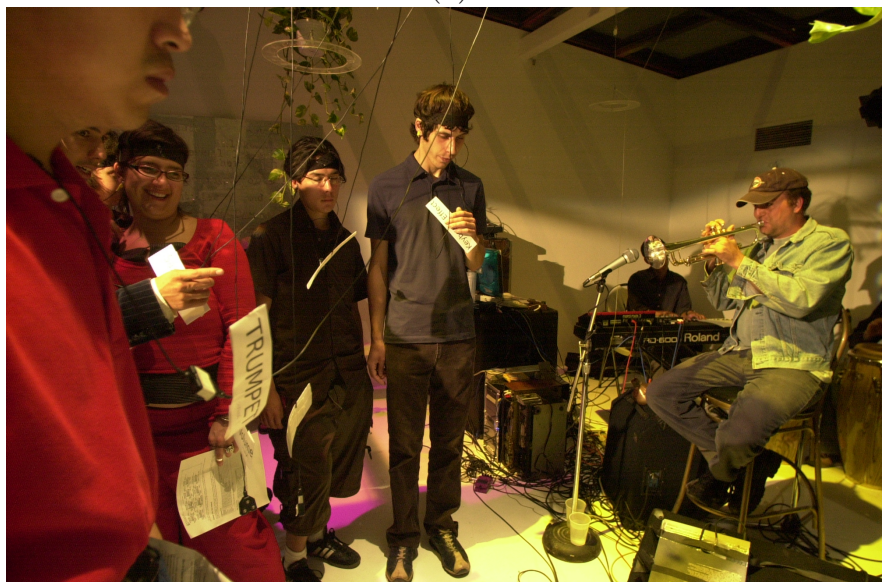

(b)

Fig. 3. Regenerative Jazz Performance (a) DECONcert 2: Audience brainwaves modulate the sounds of a trio of performers. (b) DECONcet 3: A Jazz ensemble is affected by audience brainwaves, with acoustic instruments modulated via sound filter.

\subsection{Telematic Tubs Against Terror}

Telematic Tubs Against Terror, also explored the creation of a collective and communal consconsious, this time using the mediums of water and brainwaves, rather than music and brainwaves. Figure [5 shows images taken from these events. Two tubs of water were set up in different locations, one on a main street, and one indoors 1.5 miles away. Eight EEG leads and several ECG leads were suspended over the tubs. Two screens abutted the tubs. Each location received the EEG and ECG information of the sister tub and projected it on one screen, 


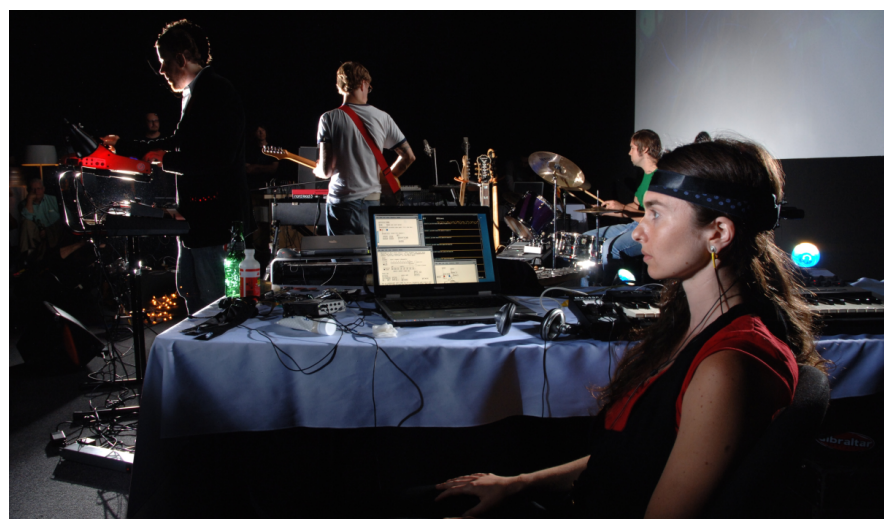

(a)

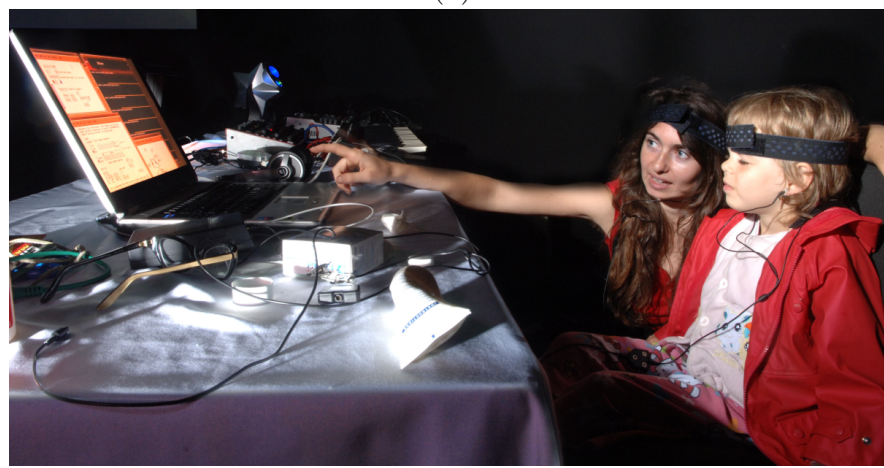

(b)

Fig. 4. Brainwave Performance at the Powerplant Art Gallery in Toronto: (a) Quintist Ariel Garten performing; (b) A young audience member performs in the concert, after a brief 5 minute training session

as well as receiving live video feed from the sister location projected onto the second screen. Up to 8 (and sometimes more) participants at a time entered the tub together, and connected themselves to the EEG and ECG leads. In this way the participants were sharing not only physical space, but mental space as well.

\subsection{Differentiating Brainstates to Create Control Interfaces}

In DECONcert 1, we hooked up 48 people's EEG signals, which were collectively used to affect the audio environment. Each audience member had a single EEG lead held against the back of his or her head with comfortable headband, at the location of the occipital lobe. As well, a wire was clipped to each ear for grounding. The collective signals from groups of eight participants were cleaned, and collective alpha synchronization (which occurs, for instance, when people close their eyes) was detected.

The alpha-wave intensity increases when a person approaches a calm meditative state of concentration and it is inversely proportional to the amount of 


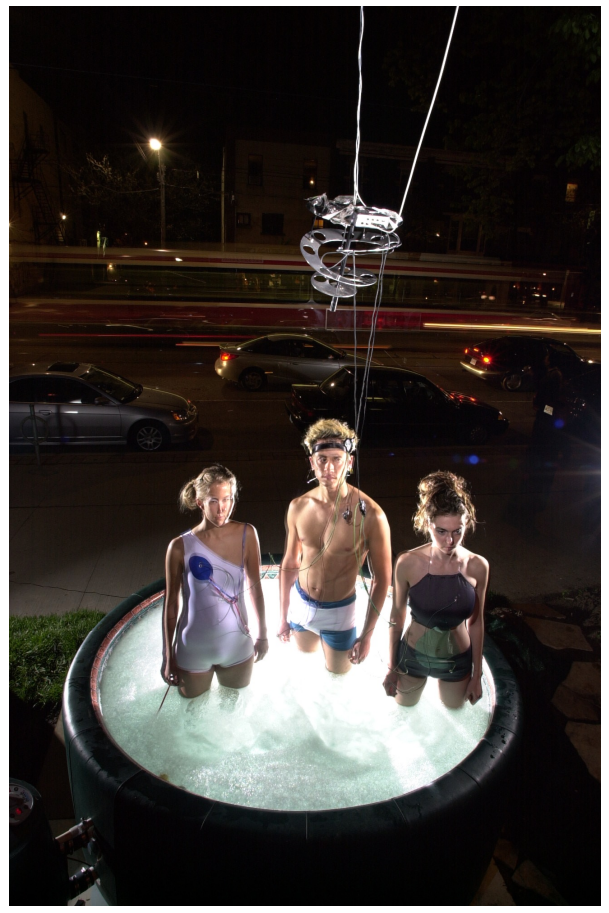

(a)

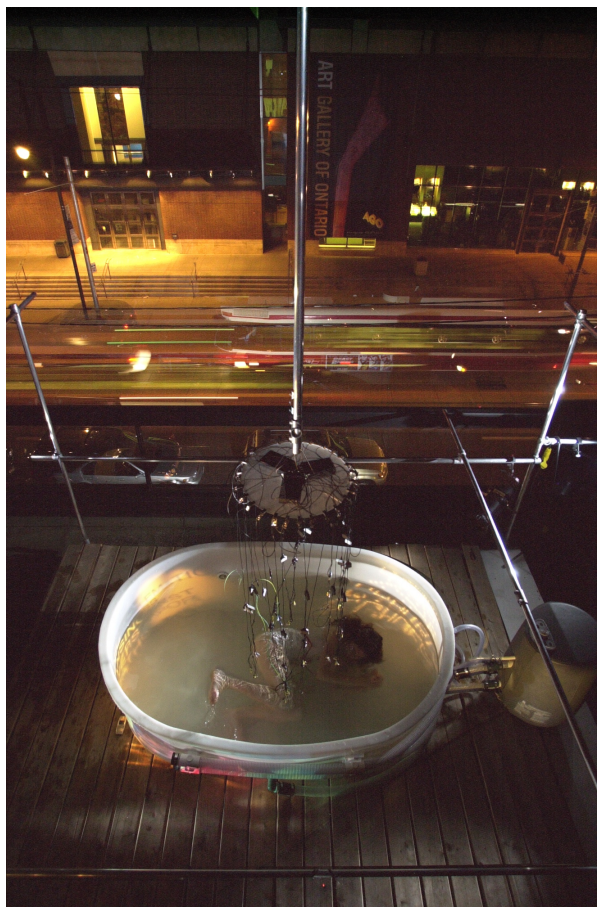

(b)

Fig. 5. Telematic Tubs Performance. (a) and (b) show two different "wash nodes" where participants' EEGs and ECGs were read and shared between sites. A video link connected each wash node with the others as well, and brainwave data was displayed and re-presented remotely.

visual stimulant the person receives 3 . Experiments have shown that there exists a correlation between the mental activity of a person and their respective EEG spectrum [2]. Lusted and Knapp explored brainwave interfaces [6]. An early musical brainwave implementation was conducted by Lucier 4 5, also employing alpha waves as a sonic device. Rosenboom [8] worked with alpha waves for music production. The music of this work extends the group dynamic of brain wave music to 48 simultaneous participants, and explores both light and water as additional mediums for immersive experiences.

Humans are generally described as being in one of 5 brain states, Alpha, a calm creative state that is described by brainwave activity of $8-12 \mathrm{~Hz}$, Delta, slow brainwave less thank $2 \mathrm{~Hz}$, is associated with deep sleep. Theta, a state achieved by those in deep meditation or earlier stages of sleep, are classed as 4-8 Hz. Most individuals spend most of their day in beta waves, classified as any wave activity over $12 \mathrm{~Hz}$. 1] For our purposes, we tracked whether participants were in Alpha state $(8-12 \mathrm{~Hz})$ or another state. 


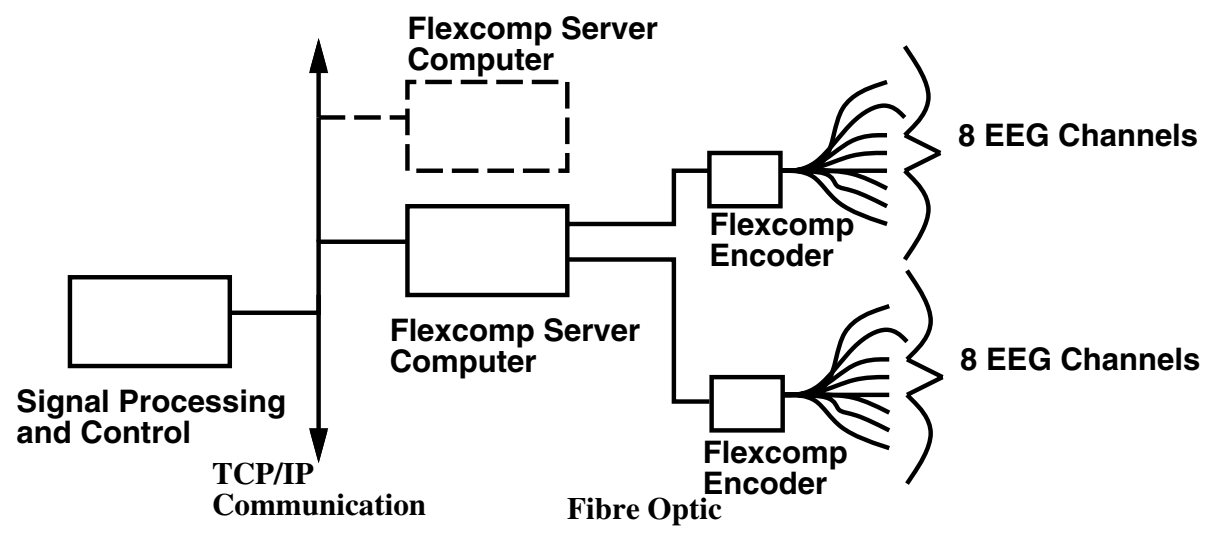

Fig. 6. EEG Multimedia Control System. The system is expandable to accommodate a number of EEG channels, which may be connected to multiple participants. Additionally, the TCP/IP connection allows the possibility of remote and wireless data analysis and storage.

System Configuration. The basic configuration of the system is shown in Figure [6] To digitize brainwave activity for analysis, a Thought Technology FlexComp A/D encoder and ISA DSP2 Data Acquisition Card (DAC) are used. These devices can provide up to a $2 \mathrm{KHz}$ brainwave sampling rate, and measure brainwave activity down to a maximum of $5 \%$ error and $1 \mathrm{~V}$ accuracy.

A set of custom programs were written to utilize the hardware for music generation. Additionally a Linux device driver was written to interface with the ISA data acquisition card. A server program communicates with the DAC, placed on the ISA bus of a Linux system, and optically connected to the FlexComp encoder hardware thereby making raw EEG data available over TCP/IP. A client system connects to the server via TCP/IP and receives the EEG data, upon which it performs the filtering and processing of brainwave data. Both programs can run on a single Linux PC using the loopback address (127.0.0.1), if the PC is sufficiently fast. Similarly the TCP/IP interface can be exploited to allow communication between remote locations, as in the implementation of the Telematic Tubs exhibit, and the remote Parkinson's patients event.

Additionally control of standard AC room lighting was achieved using a DMX512 dimmer system is used. DMX-512 is a simple packet-based digital protocol for controlling stage lighting and other devices using an RS-485 serial interface at 250kbaud connecting to a LanBox LCX DMX-512 Controller over a TCP/IP socket.which sends a single text command to change room lighting levels. This change is transmitted via the DMX protocol to a set of DMX dimmers, which change the light intensities in the room as required. Figure $\mathbf{7}$ shows the EEG controlled lighting environments used in the exhibits. 


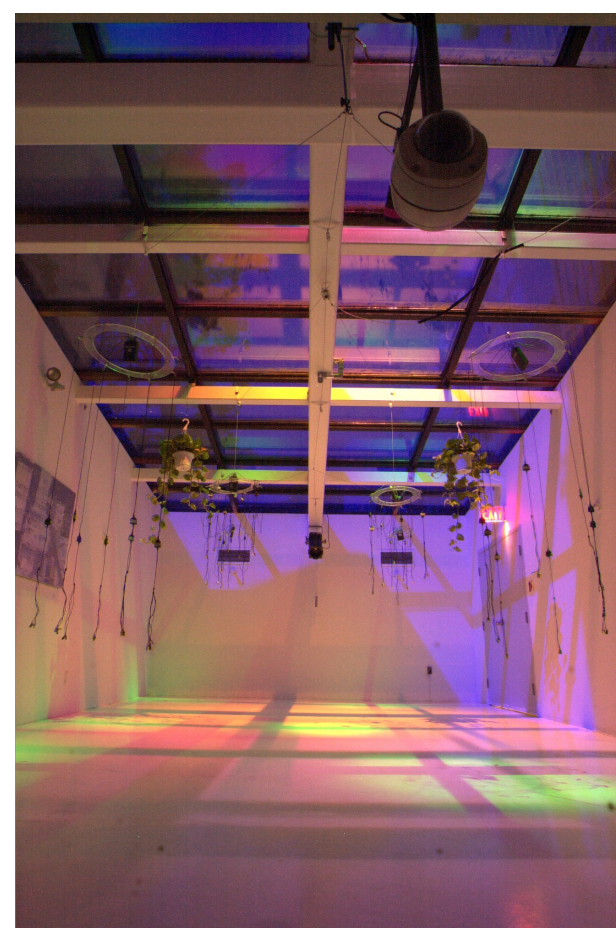

(a)

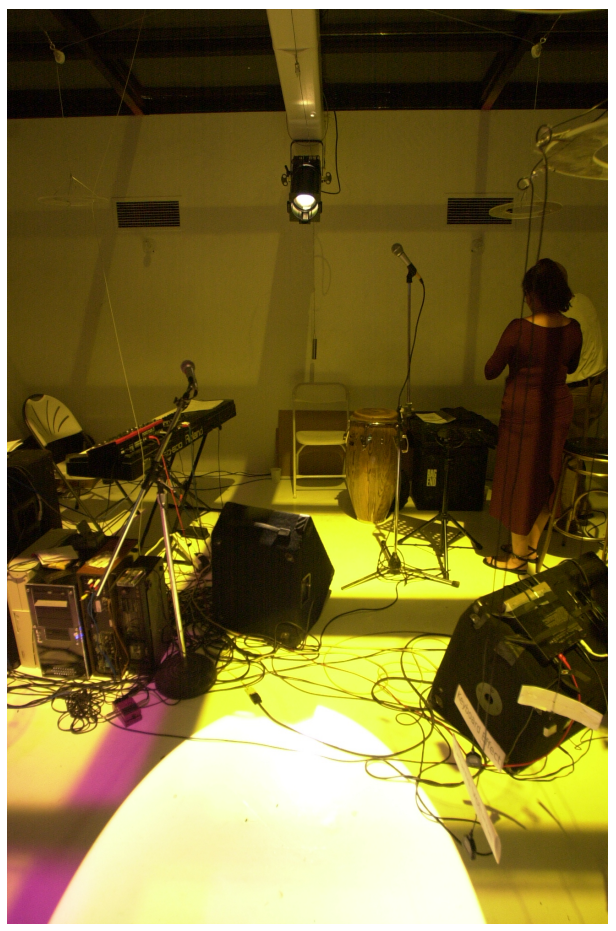

(b)

Fig. 7. Brainwave Controlled Immersive Lighting and System Sculpture. (a) The DECONcert theatre. Six stations of EEG Electrodes hang from the ceiling. Gelled lighting systems shining from above the skylights of the space use light to create a collective immersive environment. The custom designed circular discs form EEG nodes for eight participants, reflecting the "neuron" like design of the system where multiple branches of input flow to a signal processing machine. (b) In turning the audience into a participant, DECONcerts invert the relationship between audience and performer, bringing the two together. A brainwave controlled spotlight shines on the participants with the intensity controlled by their concentration state.

\subsection{Screening Out Unusable Signals}

EEG signals are typically orders of magnitude weaker than muscular signals. Consequently, if the participant is moving their head, or their muscles are not sufficiently relaxed, the EEG signal signal strength is weak in comparison to the muscular electrical activity, which we consider noise. In this situation, we cannot rely upon the analyzed EEG bands to produce a usable signal. In order to detect these cases, we calculated the power of the received signal, and rejected the signal above a certain threshold, which could be calibrated as the system was used. 


\subsection{Real-Time Control of Live Musical Input}

Because the system is used for real-time control, latency between the onset of a desired EEG trend, and response of the system was found to be of interest. We implemented several approaches to control. The first approach was to use a variable counter which incremented so long as the participant's alpha waveband strength was above a threshold. The counter decremented when the alpha waveband strength was below a threshold. Sound effects were triggered when the counter was above a certain threshold. The advantage of this method was that only sustained period of high alpha activity triggered a sound response from the system, and made it quite robust with respect to a "false positive" alpha strength detections. Additionally, this method allowed us to verify the efficacy of our system at detecting alpha activity. However, the requirement for sustained periods of alpha activity meant that the sound effects would only occur at typically longer than 10-15 seconds after the onset of the alpha activity state. This latency made it difficult for a casual participant to perceive their effect on the sound. Similarly the window sized used for the frequency analysis is related to the latency in the system. Longer windows allow for more reliable detection of sustained mental state. However, this increased overall latency of the produced control signal. Additionally, high, but short lived alpha activity is not well detected in this case. Short windows allow for faster system response, but were affected by noise.

\subsection{EEG Based Music Composition}

Our approach to EEG based musical composition was that of creating a general programming framework, whose variables were continuously controlled by the EEG signals of the participants.

There was a simple sequenced bassline (randomly choosing from 4 note progressions), a simple sequenced drum track (the complexity of the track altered by the persons alpha), which utilized a sequencer. We used the counter method described above. However, instead of only a single event above a preset threshold, different ranges of the current value was used to determine the complexity of the tracks.

For the bassline, several sets of notes' on and off toggles were under control of the EEG. Thus, for higher activity, more notes were turned on and this made the sound of the baseline appear more busy and complex. For the sequenced drum track, the EEG was used to toggle different rhythm tracks on and off. When more instruments were triggered on, the rhythm appeared more complex. Again, these were turned on and off with respect to current alpha counter range. Additionally pad and background sounds were randomly triggered by the amount of alpha activity of the participant.

To maintain a musical consistency the tones (notes) were chosen from a predetermined scale (aeolian mode) so as minimize the disonnance which would occur if completely random notes were used. For these effects, however, the primary contribution of EEG control was to affect the filter frequencies of the tones, 
which dramatically affected their quality. The control was achieved via sending MIDI control signals to synthesizers.

Typically observed minimum and maximum alpha waveband strengths were mapped to the range $[0,127]$ used by MIDI. This method represented a continuous form of mapping alpha strength to control variables as no thresholding was used. Additionally, however, we found that the most effective sound effects were those which changed dramatically over their MIDI controlled range. In some instances, we restricted the MIDI controller value into a range which produced dramatic changes instead of the full $[0,127]$.

We found that this approach allowed the system to emulate the pseudorandomness of sweeps and pads which tend to occur, for instance, in electronic music, and create those events under the control of the alpha. Similarly, the kick drum or delay effects on a drum track are additionally triggered on and of under the control of alpha waves.

Overall, we found that these approaches allowed for different parts to fade in and out. For instance, the kick sometimes provided a beat, and would then fell out, giving way to a more open segments and so on. The participants were able to learn and control the system well by listening to the music feedback, over the 30 minutes they had to use it. At the end of the performance, the participants understood their control of the music well.

\subsection{Affecting Live Performance with EEG Signals}

DECONcerts 2 \& 3 used participant brainwaves to alter the sound qualities of instruments being played by live musicians. Both acoustic and electronic instruments were affected. Electronic instrument sound qualities were affected by varying MIDI parameters using the system of DECONcert 1. An electronic keyboard, electronic wind instrument and electronic drum pad were used.

To achieve variation on acoustic instruments, a digital mixer was used. For acoustic instruments such as an amplified bass 3 , two audio channels were fed into the mixer. One channel was the unaltered audio channel, and the second was the same input run through a filter. The filter was either a pedal filter, or a digital filter applied internally: a feature of the mixer equipment. Brain wave signals drove the system to, via MIDI, crossfade between the unfiltered and filtered channels. In this way the brainwaves altered the sound quality of the acoustic instruments used at the event.

This created a challenging playing environment for the musician. The sound quality of the musician's instrument changed in ways that were not under the conscious, direct control of the musician. As the sound quality changed, the musician needed to adapt their playing style to match. For instance, the decay of the note would change. With a short decay, the musician could perform quick, stacatto phrases, while longer decays, phrases incorporating sustained notes were more appropriate. In this way, participants affected the overall qualities of the music despite the fact that the musicians were playing the instruments themselves.

${ }^{3}$ For the moment, we consider the analog nature of the amplification of the vibration of the strings of an electric bass guitar as "acoustic". 


\section{Aquatic Context: Waves in Water and Mind}

The connection between brainwaves, water waves, and sound waves (in both air and in water) was made all the more apparent in a recent performance at the International Computer Music Conference (ICMC 2007) in Copenhagen. The theme of the 2007 conference was "Immersed Music".

In keeping with this theme, we developed various forms of "Immersive Media" for public performance in Copenhagen's Vandkulturhuset. The name "Vand kultur huset" means "water culture house" in Danish.

\subsection{The States-of-Matter Quintet}

This Immsersed Music concert at ICMC 2007 consisted of a performance by the States-of-Matter Quintet, involving musical instruments we created that produced sound from each of the five states-of-matter:

- Solid ("Earth");

- Liquid ("Water");

- Gas ("Air");

- Plasma ("Fire");

- Quintessence ("Idea").

These correspond to the Greek Classical Elements, the fifth element being Idea (non-matter).

See Fig 8 .

\subsection{Surrounding Medium}

Vandkulturhuset (Fig. 91),

The existence of immersive media raises the question of media itself. Thus we may ask:

- in what medium is the sound initially produced;

- what is the surrounding medium;

- in what medium is the listener immersed?

For example, the fact that a listener may be immersed in air, or in water, suggests also that the sound need not be produced in the same medium in which it is experienced.

\section{Philosophical Implications/ Discussion}

\subsection{Human/Computer Feedback Interaction (HI)}

Both Telematic Tubs Against Terror and DECONcert create a Humanistic Intelligence feed-back loop using the elements of music, water and brain. As discussed, Humanistic Intelligence is defined as intelligence that arises from the 
human being in the feedback loop of a computational process in which the human and computer are inextricably intertwined, in otherwords, it is a regenerative feedback loop. In DECONcert, regenerative music is the expression of $\mathrm{HI}$ in music. In regenerative music the computer, instead of taking only active cues from the musician, reads physiological signals from the musician/performer. The music which the regenerative algorithm then creates will be heard by the musician/performer. It is hoped that the music will in turn generate an emotional response on the part of the musician/performer, and that that emotional response will be detectable by the computer, which can then alter the music in some way in response. Continuing in this fashion, it is clear that there is a well defined feedback loop occurring between the human and the computer.

\subsection{Regenerative Music}

Jazz, the musical genre of the DECONcert performances, is a natural noncomputation example of regenerative music. Jazz is a free flowing style of musical improvisation in which the performers intuitively read one another's states, mood and musical intention based on both the performer's sonic output as well as conscious and subconscious communication between players. The players in a sense create an immersive, responsive environment. Immersed in the music that surrounds them, in the musical and collective 'zone', they respond to one another's output. The response of the audience, also encourages or discourages the musicians' particular output. DECONcert takes this intuitive process and turns it inside out.

Regenerative Music looks not only at the audience-musician interaction, but also the musician-instrument response. It brings in the problem of how a musician can learn to respond to this new physiologically driven instrument, as well as how the instrument can learn to infer the wishes of the musician through their physiological signals, in addition to the normal playing of the instrument. In a sense, the musician and instrument each play off of each other, and together, both can be viewed as an "instrument". The choice of how to map from physiological signals into instrument behavior would be an artistic one, under the control of the musician.

\subsection{Collective Unconsciousness}

Creating regenerative music then becomes a distributed process, where no one individual has conscious control over the sound. In a sense, all individuals enter a collective state in which no single individual is aware of or has conscious control over the outcome. Collectively and communally, the audience determines what will be heard musically, using the interface of their brains. The audience has no determination over what the final outcome of the music will be, nor do the musicians. Thus, with brains physically connected to one another though EEG leads, the audience enters both physically and metaphorically into a collective unconsciousness.

In Telematic Tubs Against Terror, collective unconsciousness was explored not through music as its medium of expression, but through water. Sitting together 

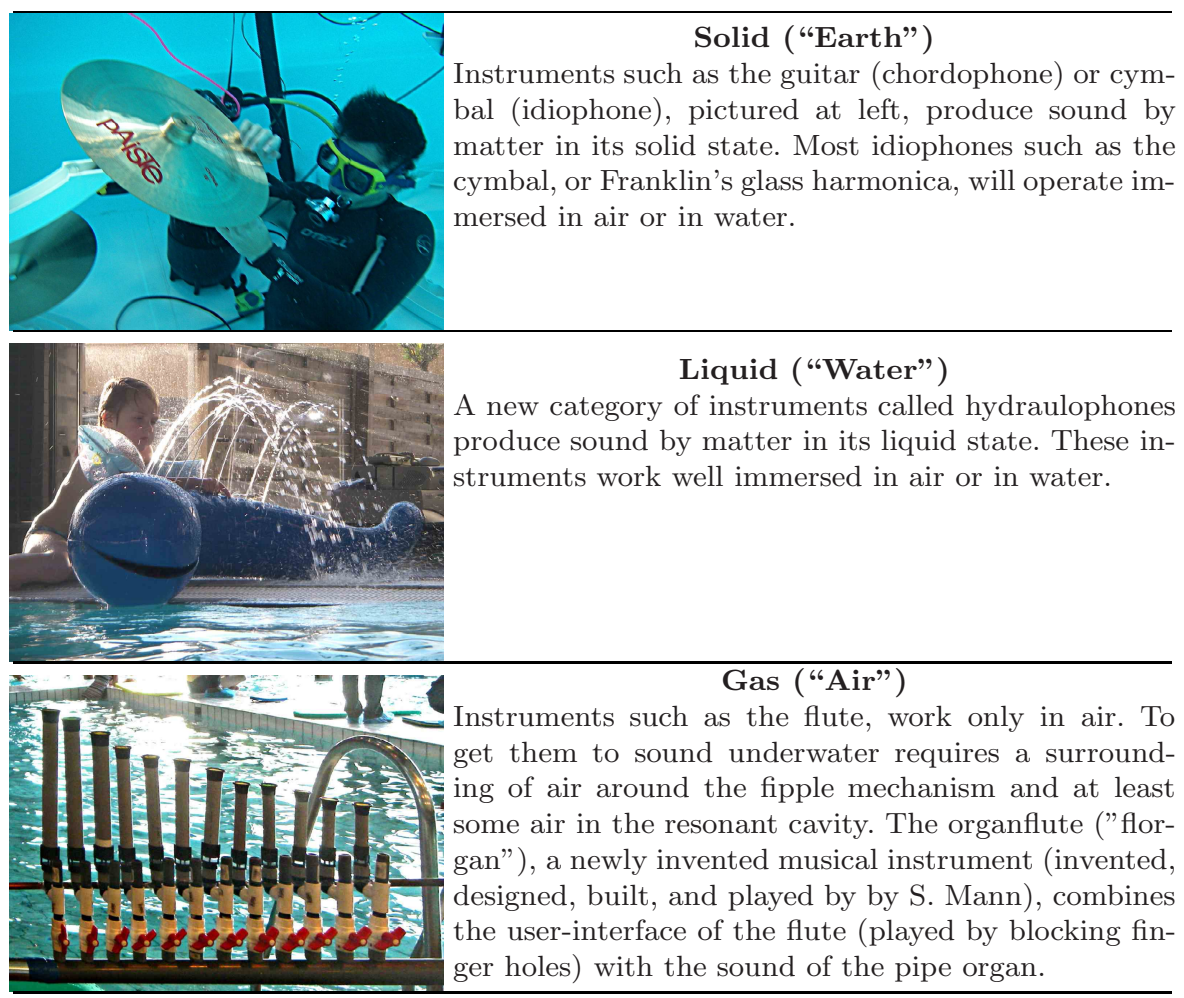

Liquid ("Water")

A new category of instruments called hydraulophones produce sound by matter in its liquid state. These instruments work well immersed in air or in water.

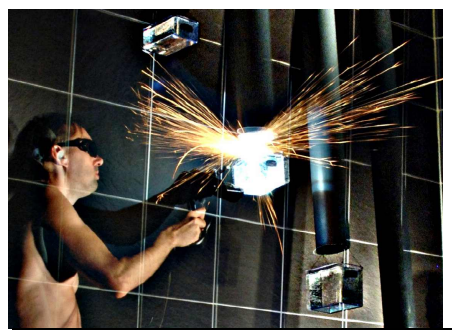

\section{Gas ("Air")}

Instruments such as the flute, work only in air. To get them to sound underwater requires a surrounding of air around the fipple mechanism and at least some air in the resonant cavity. The organflute ("florgan"), a newly invented musical instrument (invented, designed, built, and played by by S. Mann), combines the user-interface of the flute (played by blocking finger holes) with the sound of the pipe organ.

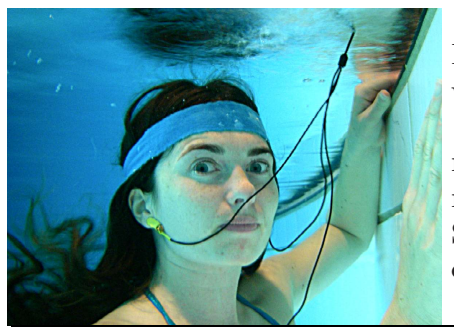

\section{Informatics ("Idea")}

Plato and Aristotle postulated a fifth state-of-matter, which they called "Idea" or "Quintessence" (from "quint" which means "fifth"). This covers thoughts, mathematics, algorithms, and the like. A direct brainmachine interface was used in the performance by the States-of-Matter Quintet to represent the fifth stateof-matter.

Fig. 8. Five States of Matter in Immersive Music. These five states-of-matter correspond to the five Classical Greek Elements. Earth, Water, Air, Fire, and Idea ("Quintessence" meaning "fifth" element). Immersed Music concerts explored the immersion of these five elements in both water and air. Immersed Music was the theme of ICMC 2007, which included concerts at the DGI-byen "Vandkulturhuset" swim center. 


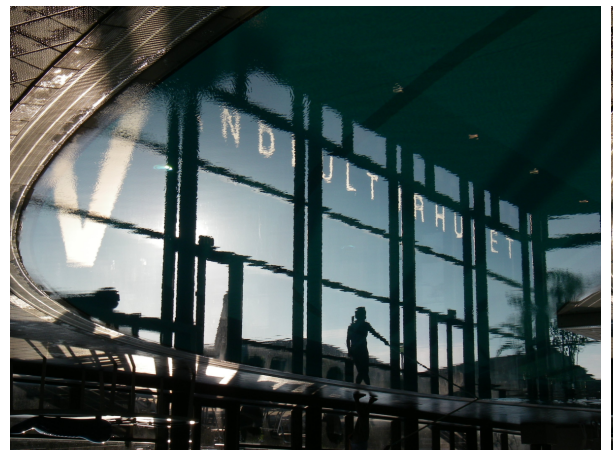

(a)

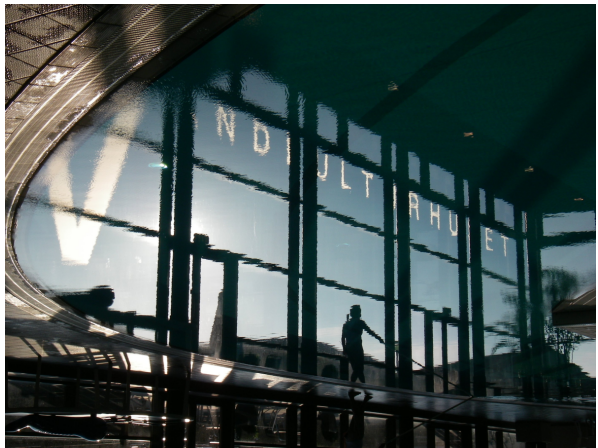

(b)

Fig. 9. "Vandkulturhuset" is Danish for "Water Culture House". This cultural venue in Copenhagen was the site of our "Immersed Music" concert.

in a pool of water collecting and sharing brainwave information, participants were brought into the same collective DECONsciousness, as DECONcert. In also known as the DGI Swim Centre, consists of six pools in the main DGI-byen aquatics area, which provide a variety of different kinds of bathing experiences at a variety of different temperatures. One of these pools is a 100 metre (320 foot) round pool called "the ocean". The ocean pool allows for "endless" (no need to stop and change direction) swims. This round pool also has a hydraulic stage in the center for concerts or banquets, in which the round pool forms a moat around the musicians or diners. Our performance actually brought a variety of musical instruments right into the pool itself, creating truly immersive music! See Fig. [10]

\subsection{Sousveillance}

Surveillance pervades our society, ostensibly to mitigate danger. As a collective of individuals whose information is being shared amongst one another, we are engaging in a "sousveillance" [9] of sorts. Whereas traditional surveillance is a top-down affair, where some hierarchically superior "Big Brother" is watching the movements of the general populace, in Telematic Tubs Against Terror and DECONcert, the distributed sharing of private information creates a sousveillance, a situation in which the subjects themselves are recording and sharing their own information with one another. In an interesting twist, this sousveillance creates a feedback loop between the audience and the recording device, particularly the complex feedback loop in Telematic Tubs Against Terror between the video cameras and 2 separate wash nodes. In being recorded, particularly while in the vulnerable situation of bathing, one's behavior changes. In Telematic Tubs, one realizes one is being recorded, and ones actions are affected as such. Those different reactions are broadcast to the sister tub, who in seeing the actions of their fellow bathers at a separate location, act differently. These 


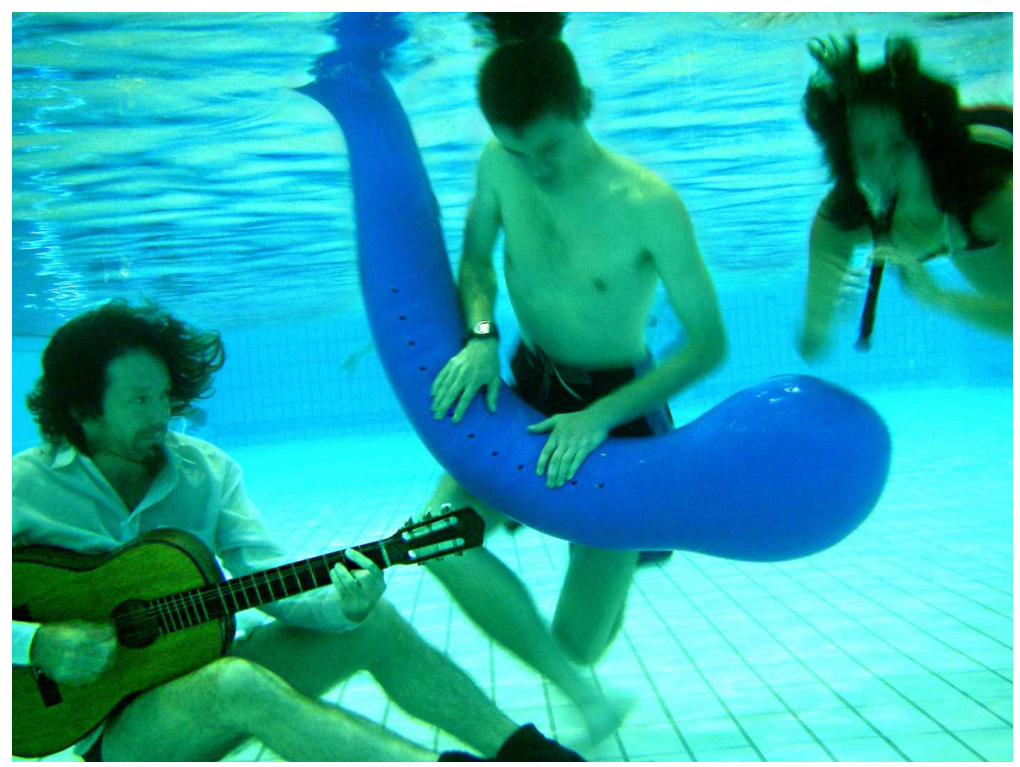

Fig. 10. Instruments that make sound from solid matter (left), liquid matter (middle) and gaseous matter (right). Here instruments making sound from all three states-ofmatter (solid, liquid, and gas) are immersed in a surrounding medium of liquid. Only the hydraulophone is designed to work properly when immersed in liquid.

reactions of the participants in the second tub are then recorded on video projected on a screen by the first tub, to further effect the behavior of the participants there, who cannot directly view on a screen their own behavior, only that of the second tub. Thus a self-conscious distributed self-surveillance feedback loop is created.

\section{Conclusion}

DECONcert and Telematic Tubs Against Terror speak of the interconnected relationships between water, music and the brain. Using an intelligent signal processing system to control musical output we created a collective consciousness by highlighting both the humanistic intelligence human-computational feedback loop as well as a physical shared immersive environment. In creating what we refer to as a collective DECONsciousness, issues of privacy, contamination, and control become significant. Participants become performers who are on stage in the bath, self powering a distributed immersive experience with the pooling of their brainwaves.

\section{Acknowledgements}

The authors would like to acknowledge the contributions from Corey Manders, Chris Aimone, and Ryan Janzen. We also thank Thought Technologies Limited, 
www.thoughttechnology.com, for donation of equipment that made much of this work possible.

\section{References}

1. Brankack, J., Stewart, S.F.M.: Current source density analysis of the hippocampal theta rhythm: Associated sustained potentials and candidate synaptic generators. Brain Research 615(2), 310-327 (1993)

2. Cromwell, L., Weibell, F.J., Pfeiffer, E.A., Usselman, L.B.: Biomedical Instrumentation and Measurements. Prentice Hall, Inc., Englewood Cliffs (1973)

3. Legewie, H., Simonova, O., Crutzfeldt, O.D.: EEG Changes During Performance of Various Tasks Under Open- and Closed-eyed Conditions. Elsevier Publishing Company, Amsterdam (1969)

4. Lucier, A.: Music for Solo Performer (1965), for enormously amplified brain waves and percussion. Lovely Music, Ltd., (1982)

5. Lucier, A.: Music for alpha waves, assorted percussion, and automated coded relays, on "imaginary landscapes". Elektra/Nonesuch 79235-2 (1989)

6. Lusted, H.S., Knapp, R.B.: Controlling computers with neural signals. Scientific American Magazine, 82-88 (October 1996)

7. Mann, S.: Humanistic intelligence/humanistic computing: 'wearcomp' as a new framework for intelligent signal processing. Proceedings of the IEEE 86(11), 21232151 (1998), http://wearcam.org/procieee.htm

8. Rosenboom, D.: Method of producing sounds or light flashes with alpha brain waves for artistic purposes. Leonardo 5(1) (1972)

9. Steve Mann, M.F., Fung, J., Baccanico, G.: Panopdecon: deconstructing, decontaminating, and decontextualizing panopticism in the postcyborg era. Surveillance \& Society 1(3), 375-398 (2003) 


\section{Author Index}

Afanador, Kathleya $\quad 34,235$

Amatriain, Xavier 380

Aramaki, Mitsuko 139

Ardila, Mauricio 125

Avanzini, Federico 212

Baalman, Marije A.J. 246

Barthet, Mathieu 313

Burgoyne, John Ashley 181

Campana, Ellen 34, 235

Carter, Jenny 74

Castellanos, Jorge 380

Chordia, Parag 110

Chowning, John 1

Dannenberg, Roger B. 276

Dean, Roger 223

de Götzen, Amalia 212

Driessen, Peter F. 276

Eaglestone, Barry 74

Eigenfeldt, Arne 368

Einbond, Aaron 203

Ellis, Jarrod 453

Fabiani, Marco 288

Ford, Nigel 74

Friberg, Anders 288

Fung, James 487

Garten, Ariel 487

Gerhard, David 453

Godfrey, Mark 351

Haines, William D. 276

Hamilton, Robert 478

Holdridge, Peter 74

Höllerer, Tobias 380

Ilomäki, Tuukka 98

Ingalls, Todd 34,235

James, Jodi 34, 235

Jensen, Kristoffer 263
Kalayar Khine, Swe Zin 159

Kendall, Gary S. 125

Knapp, R. Benjamin 441

Kobayashi, Ryoho 360

Kronland-Martinet, Richard 139, 313

Kuchera-Morin, JoAnn 380

Kühl, Ole 263

Leistikow, Randal 34

Li, Haizhou 159

Lockhart, Adam 172

MacCallum, John 203

Malloch, Joseph 401

Mann, Steve 487

McAdams, Stephen 181

Merer, Adrien 139

Mion, Luca 212

Moody-Grigsby, Daniel 246

Mumford, Jessica 235

Nwe, Tin Lay 159

Park, Brett 453

Pastuszek-Lipińska, Barbara 56

Pérez, Miguel Angel Ortiz 441

Pope, Stephen T. 380

Qian, Gang 235

Rae, Alex 110, 351

Rajko, StJepan 34, 235

Rath, Matthias 303

Rhoads, John 351

Salter, Christopher L. 246

Serafin, Stefania 212

Sinclair, Stephen 401

Stevens, Catherine 223

Swaminathan, Dilip 34, 235

Takahashi, Masato 360

Taylor, J. Milo 426

Thornburg, Harvey 34, 235

Tillmann, Barbara 11 